\title{
3D direct writing of bandgap tunable nanocrystals in transparent material
}

\author{
Feng Chen \\ Shandong University, Shandong, China
}

Bandgap tuning of semiconductor nanocrystals is an effective way to create new functional materials with tunable properties, which is critical for realizing many photonic applications, such as light-emitting diodes, lasers, and photodetectors. ${ }^{1}$ Several synthetic, processing, and stabilization steps are currently necessary and organic contamination is introduced.

Incorporating nanocrystals endows transparent materials (e.g., glasses) with advanced optical functionalities and access to plenty of applications. ${ }^{2}$ However, it is a great challenge to three-dimensionally tailor the chemical composition of nanocrystals inside the glass matrix. In fact, manipulating the bandgap by changing the chemical composition at the nanoscale in solids is always full of challenges. Recently, ultrafast laser direct writing offers an intriguing technique to create 3D functional micro- and nanostructures in transparent solids, however, the composition tunability has been limited.

A group led by Jianrong Qiu and Dezhi Tan has reported a unique strategy of ultrafast-laser direct lithography for engineering the local chemistry at the nanoscale in glass, to achieve three-dimensional direct writing of nanocrystals with a widely tunable bandgap (Fig. 1). ${ }^{4}$ They revealed a fundamentally new mechanism of ultrafast laser-matter interaction. Specifically, ultrafast-laser pulses inject energy within an ultrashort time interval and cause strong thermal accumulation. Consequently, the pressure and temperature increase, leading to melting of the glass matrix and localized liquid nanophase separation. After shuttering the laser pulses, the nanophase domains will crystallize as nanocrystals. This research has led to major breakthroughs in research about glass, perovskites, ultrafast-laser-matter interaction, and ultrafast-laser manufacturing.

By controlling the laser parameters and irradiation, they modulated the local conditions and the resultant chemical compositions of the nanocrystals via ultrafast-laser-induced liquid nanophase separation and the site exchange of ions in glass. Taking perovskite nanocrystals as an example, they successfully realized the transformation of perovskite nanocrystals from $\mathrm{CsPb}\left(\mathrm{Cl}_{1-x} \mathrm{Br}_{\mathrm{x}}\right)_{3}$ to $\mathrm{CsPbI}_{3}$ in a single glass chip and tuned the bandgap of perovskite nanocrystals with the photoluminescence between 480- and 700-nm wavelengths.

It is worth noting that perovskites exhibit high sensitivity to polar solvents, oxygen, and light, and their low structural stability is a great obstacle for the applications of perovskite devices. ${ }^{5}$ In this work, the perovskite nanocrystals are encapsulated in the glass matrix which keeps them away from different molecules in the environment; as a result, the nanocrystals exhibit improved stability against ultraviolet light irradiation, organic solutions, or temperatures up to $250^{\circ} \mathrm{C}$.

From the device standpoint, the patterning is important for nanocrystal-based device fabrication; device manufacture has been complicated with multistep processing. ${ }^{6}$ Composition-tunable nanocrystal patterns are written directly in a single step in this new strategy, without using any organic molecules. More importantly, multiple applications

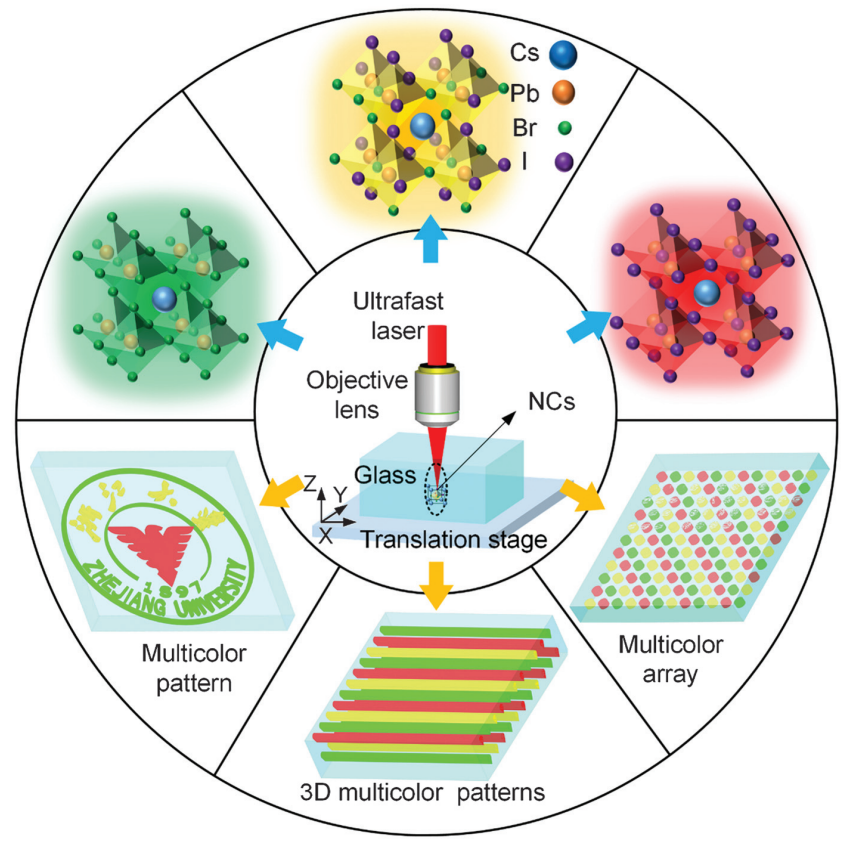

Fig. 1 Ultrafast laser direct lithography of perovskite nanocrystals and patterns with tunable compositions and photoluminescence in glass. Credit: Jianrong Qiu, Zhejiang University.

are proposed and demonstrated, such as 3D optical storage, micro-lightemitting diodes, and holographic displays.

\section{References}

1. L. Protesescu et al., "Nanocrystals of cesium lead halide perovskites $\left(\mathrm{CsPb}_{3}, \mathrm{X}=\mathrm{Cl}, \mathrm{Br}\right.$, and I): novel optoelectronic materials showing bright emission with wide color gamut," Nano Lett. 15(6), 3692-3696 (2015).

2. A. Llordés et al., "Tunable near-infrared and visible-light transmittance in nanocrystal-in-glass composites," Nature 500(7462), 323326 (2013).

3. D. Tan et al., "Photonic circuits written by femtosecond laser in glass: improved fabrication and recent progress in photonic devices," Adv. Photonics 3(2), 024002 (2021).

4. K. Sun et al., "Three-dimensional direct lithography of stable perovskite nanocrystals in glass," Science 375(6578), 307-310 (2022).

5. Y. Wei et al., "An overview on enhancing the stability of lead halide perovskite quantum dots and their applications in phosphorconverted LEDs," Chem. Soc. Rev. 48(1), 310-350 (2019).

6. Z. Liu et al., "Micro-light-emitting diodes with quantum dots in display technology," Light Sci. Appl. 9(1), 83 (2020).

(C) The Authors. Published by SPIE and CLP under a Creative Commons Attribution 4.0 International License. Distribution or reproduction of this work in whole or in part requires full attribution of the original publication, including its DOI.. [DOI: 10.1117/1 AP.4.1.010502] 